\title{
Пимекролимус в лечении атопического дерматита у детей. Вопросы безопасности и эффективности. Опыт пятилетнего применения
}

\author{
Д.В. Заславский
}

ГБОУ ВПО «Санкт-Петербургский государственный педиатрический медицинский университет» Минздрава России

194100, Санкт-Петербург, ул. Литовская, д. 2

В последнее десятилетие отмечается рост заболеваемости атопическим дерматитом (АД) у детей грудного возраста. Раннее формирование АД (в возрасте 3-6 мес.) отмечается у 45\% детей, в течение первого года жизни - у $60 \%$. K 7 годам у 65\% детей, а к 16 годам у 74\% детей с АД наблюдается спонтанная ремиссия заболевания. У 20-43\% детей с АД в последующем развивается бронхиальная астма и в 2 раза чаще — аллергический ринит. Несмотря на то что топические кортикостероиды (ТКС) назначаются достаточно часто, существует необходимость в негормональной терапии из-за слабой приверженности лечению ТКС ввиду побочных эффекктов. Течение АД нередко осложняется колонизацией золотистым стафилококком и неэффективностью наружной терапии топическими кортикостероидами, так как у большинства пациентов с АД обнаруживается высокий уровень IgЕ против суперантигена стафилококка. В длительном и крупномасштабном исследовании, проведенном с участием детей младшего возраста с АД легкой и средней степени тяжести, сравнивали пимекролимус 1\% крем (ПИМ) и ТКС.

Материал и методы. В пятилетнее открытое исследование было включено 2418 детей, которых рандомизировали на группы получавших ПИМ ( $n=1205$; при обострении — краткосрочное применение ТКС) или ТKС $(n=1213)$.

Главной целью исследования было сравнить безопасность двух методов лечения; дополнительная цель заключалась в подтверждении долгосрочной эффективности ПИМ. Лечение считали успешным, если достигалась оценка по шкале IGA (Глобальная оценка исследователя) 0 (чистая кожа) или 1 (почти чистая).

Результаты. Действие в обеих группах препаратов — ПИМ и ТKС — наступало быстро, к 3-й неделе успешность лечения констатировали у > 50\% пациентов. В обеих группах после 5 лет лечение оценивалось как успешное у > 85\% пациентов, а лечение проявлений АД на лице было эфрфективным у 95\% испытуемых. В группе ПИМ потребность в стероидах была существенно ниже, чем в группе TKC (7 дней применения против 178 дней в группе ТKС). В обеих группах были сходные по характеру и частоте нежелательные явления и не выявлено нарушений гуморального или клеточного иммунитета.

Выводы. Длительное применение ПИМ при АД легкой/средней степени тяжести у детей оказалось безопасным и не влияло на иммунную систему. При применении ПИМ удавалось существенно снизить потребность в кортикостероидах (стероидсберегающий эффект). Полученные данные свидетельствуют о том, что ПИМ так же эффективен, как ТКС, и его можно примененять в качестве первой линии терапии АД легкой и средней степени тяжести у детей грудного и младшего детского возраста.

Ключевые слова: атопический дерматит, лечение и профилактика атопического дерматита. 


\title{
Pimecrolimus in atopic dermatitis in children. Safety and efficiency of 5-years treatment
}

\author{
D.V. Zaslavsky
}

St. Petersburg State Pediatric Medical University

Litovskaya str., 2, St. Petersburg, 194100, Russia

\begin{abstract}
A growth in the incidence rate for atopic dermatitis (AD) in infants has been observed for this decade. Early formation of AD (at the age of 3-6 months) is observed in $45 \%$ of infants, and in $60 \%$ of infants - during the first year of life. As many as $65 \%$ of children (by the age of 7 ) and $74 \%$ of children (by the age of 16 ) suffering from AD have a spontaneous remission of the disease. As many as $20-43 \%$ of children suffering from AD further develop bronchial asthma, and the incidence rate of allergic rhinitis is twice as high. In spite of the fact that topical corticosteroids (TCS) are prescribed frequently enough, there is a need in the non-hormonal therapy due to poor compliance with the TCS treatment regimen in connection with side effects. Treatment of $A D$ is often complicated by the colonization with Staphylococcus aureus and inefficacy of the external therapy with topical corticosteroids because many patients with AD have a high level of IgE against the superantigen of Staphylococcus. 1\% pimecrolimus cream (PIM) and TCS were compared in a long-term large-scale study involving younger children suffering from mild to moderate AD.

Materials and methods. The five-year open-label study involved 2,418 children, who were randomized into groups receiving PIM ( $n=1205$; in case of an aggravation — short-term administration of TCS) or TCS $(n=1213)$.

The main goal of the study was to compare the safety of these two methods of treatment; an auxiliary goal was to confirm the long-term efficacy of PIM. Treatment was considered to be successful if the score based on the IGA scale was 0 (clean skin) or 1 (almost clean).

Results. The effect in both groups of the drugs — PIM and TCS — was fast, and the success of treatment was recorded for $>50 \%$ of patients by Week 3 . In both groups, treatment was determined to be successful after 5 years in $>85 \%$ of patients, and treatment of manifestations of AD on the face was efficient in $95 \%$ of all subjects. In the PIM group, the need in steroids was considerably lower than in the TCS group (7 days of administration vs. 178 days in the TCS group). In both groups, there were adverse events similar by their nature and frequency, and no disorders of humoral or cellular immunity were revealed. Conclusions. The long-term administration of PIM in case of mild to moderate AD in children was revealed to be safe and had no effect on the immune system. When PIM was used, the need in corticosteroids was considerably reduced (steroidpreserving effect). These data confirm that PIM is as efficient as TCS and can be used as the first-line therapy for mild to moderate AD in infants and younger children.
\end{abstract}

Key words: atopic dermatitis.

Corresponding author: venerology@gmail.com. Vestnik Dermatologii i Venerologii 2015; 4: 119_125.

Атопический дерматит (АД) - мультифракториальное воспалительное заболевание кожи, характеризующееся зудом, хроническим рецидивирующим течением и возрастными особенностями локализации и морфологии очагов поражения [1]. АД страдают до $25 \%$ детей младшего возраста, АД существенно влияет на показатели качества жизни как самих пациентов, так и их семей [2-5]. Согласно статистическим данным, заболеваемость АД в Российской Федерации в 2014 г. составила 234,4 на 100000 населения, а заболеваемость болезнями кожи детей в возрасте 0-14 лет - 1025,9 на 100000 соответствующего населения. Заболеваемость АД в РФ за последние 16 лет возросла в 2,1 раза. Распространенность АД среди детского населения составляет до $20 \%$, а среди взрослого населения $-1-3 \%$ [6]. АД наряду с пищевой аллергией считают начальной стадией «атопического марша", который при продолжающемся воздействии аллергена на кожу в конечном итоге приводит к развитию бронхиальной астмы и аллергического 
риноконъюнктивита [7]. В основе развития АД лежит генетически детерминированная особенность иммунного ответа организма. Характерной чертой такого генотипа является поляризация иммунного ответа в пользу преобладания и преимущественного активирования Th2 лимфоцитов, что сопровождается высоким уровнем ИЛ-4, ИЛ-5 и общего IgE. При этом отмечается снижение продукции $\gamma$-интерферона, который модулирует иммунный ответ и подавляет рост кератиноцитов. Он может тормозить синтез IgE и стимулировать синтез защитных антител. Однако воспалительные изменения в коже при АД могут развиваться и без участия $\mathrm{IgE}$, тем более что, по данным литературы, приблизительно у 25\% больных уровень IgE не превышает нормальный. Иммунные нарушения объясняют две важные клинические черты АД: гиперчувствительность кожи к ряду антигенных стимулов и снижение резистентности кожного барьера к патогенным микроорганизмам с развитием вторичных микробных и вирусных осложнений [8]. Хроническое течение АД поддерживает и зуд, который является постоянным симптомом заболевания. Кератиноциты, повреждаемые при расчесывании кожи, высвобождают цитокины и медиаторы, которые привлекают иммунокомпетентные клетки в места кожного воспаления [8]. Среди факторов, поддерживающих хроническое течение АД, следует отметить патологию органов желудочно-кишечного тракта, выявляемую у 80-97\% больных АД, хронические очаги инфекции (50-60\%), аллергические заболевания органов дыхания (30-40\%) [8].

Пациенты с АД часто нуждаются в терапевтических вмешательствах во время обострений, а иногда им требуется долгосрочная непрерывная терапия для подавления воспаления кожи. В качестве препаратов первой линии часто применяют топические кортикостероиды (TKC) [7], хотя их долгосрочная безопасность и эффрективность у детей младшего возраста не исследована. Более $80 \%$ пациентов или их родителей испытывают в связи с этим опасения, а приблизительно 1/3 пациентов с АД не соблюдают инструкции по применению ТКС из-за возможных побочных эффектов [9], что подчеркивает важность альтернативных средств для лечения АД. Пимекролимус 1\% крем (ПИМ) - топический ингибитор кальциневрина, который селективно подавляет активацию Т-лимфоцитов и тучных клеток $[10,11]$. ПИМ часто рекомендуют для нанесения на чувствительные участки кожи, так как он не нарушает барьерную фрункцию эпидермиса и не вызывает атрофию кожи [7, 12, 13]. ПИМ эфффективен и хорошо переносится детьми моложе 2 лет с легкой или средней степенью АД [14-19]. В исследовании Petite сравнивали безопасность и эффрективность ПИМ и ТКС для лечения АД легкой/средней степени тяжести у детей в течение 5 лет. С учетом всех опасений относительно влияния ПИМ на развивающуюся иммунную систему проведена оценка безопасности по результатам ряда иммунологических тестов. Благодаря дизайну исследование приближено к условиям реальной клинической практики: ТКС применяли по стандартной инструкции, а родители детей, рандомизированных в группу ПИМ, имели возможность краткосрочного применения ТKС, если использование ПИМ было недостаточным для контроля симптомов при обострениях АД.

\section{Дизайн исследования}

Главной целью этого пятилетнего многоцентрового открытого рандомизированного исследования с параллельными группами (на сайте www. clinicaltrials.gov зарегистрировано под номером NCT00120523) было сравнение безопасности ПИМ и ТКС по частоте и тяжести нежелательных явлений (НЯ) и влиянию на развивающуюся иммунную систему и скорость роста на протяжении первых 5-6 лет жизни [20]. Дополнительной целью была оценка долгосрочной эффективности ПИМ. Пациентов рандомизировали в соотношении 1:1 на группы получавших ПИМ 1\% крем или ТКС (низкой, например гидрокортизон 1\%, или умеренной активности, например гидрокортизона бутират 0,1\%; крем/мазь применяли согласно утвержденной в стране инструкции). Тот или иной ТКС назначался на усмотрение врача-исследователя. Рандомизация проводилась после стратификации по исследовательским центрам и возрастным группам (3-6 и 6-12 мес. жизни). Исследуемый препарат начинали применять сразу после рандомизации и продолжали до полного исчезновения АД или в течение всего допустимого срока применения, указанного в инструкции к конкретному препарату ТКС. Применение препарата возобновляли при появлении первых признаков и симптомов обострения АД. Исследователи объясняли родителям пациентов обеих групп признаки обострения заболевания, а родителей пациентов группы ПИМ инструктировали, когда прекращать использование ПИМ и начинать применение ТKC, т.е. когда переходить на применение «скоропомощного» препарата при обострении, не контролируемом ПИМ.

\section{Оценки эфффективности и безопасности}

Эффективность лечения оценивали врачи-исследователи при визитах пациентов в клинику по шкале IGA (0-5). Успешность лечения констатировали при оценке 0 (чистая кожа) или 1 (почти чистая); также определяли, какой процент от общей площади поверхности тела (TBSA) поражен воспалением. Особое внимание обращали на проявления АД на лице.

На каждом визите измеряли рост и массу тела. В группе ПИМ зарегистрированные НЯ могли возникнуть или во время лечения ПИМ, или во время применения ТKC при обострении. Иммунологическое обследование включало определение титров антител к стандартным вакцинным антигенам, оценку гумо- 
рального и клеточного иммунного ответа и фрнкциональные тесты для оценки Т-клеточного иммунитета.

\section{Результаты}

Всего 2439 детей были включены в рандомизированное исследование, 2418 из них получили хотя бы одну дозу исследуемого препарата (ПИМ - 1205 пациентов; ТКС - 1213 пациентов) и были включены в оценки эфффективности и безопасности. Полный курс лечения был проведен 69,4\% пациентов из группы ПИМ и 72,1\% пациентов из группы ТКС. Пациенты из обеих групп были сопоставимы по исходным демографическим и клиническим характеристикам. При включении в исследование большинство пациентов были в возрасте от 6 до $12 \mathrm{мес.} \mathrm{Хотя} \mathrm{по} \mathrm{протоко-}$ лу в исследование должны были набирать пациентов в возрасте от 3 до 12 мес., 15 пациентов были старше 12 мес. (14 пациентов < 12,5 мес.; 1 пациенту было 12,8 мес.). Популяция пациентов, прошедших иммунологическое обследование (383 из группы ПИМ и 391 из группы ТKC), имела такие же исходные характеристики, как и общая популяция (данные не приводятся).

Эффрективность ПИМ и TKС характеризовалась быстрым началом действия. К 3-й неделе был достигнут успех (т.е. IGA $\leq 1)$ у более $50 \%$ детей в обеих группах (в группе ПИМ - у 52,6\%; в группе ТKС у 50,5\%); состояние кожи на лице соответствовало критерию успешного лечения (IGA $\leq 1)$ у $61,0 \%$ в группе ПИМ и у $61,8 \%$ в группе ТKC. Медианная площадь поражения уменьшилась к 3-й неделе лечения с исходных 16\% ТВSА до < 5\% (ПИМ - 3,8\%; ТKС - 4,0\%) (рисунок).

К концу пятилетнего исследования констатировали успешное лечение (IGA $\leq 1)$ для всей кожи у > 85\% пациентов (ПИМ - 88,7\%; ТКС - 92,3\%) и у > 95\% (ПИМ - 96,6\%; ТКС - 97,2\%) успешное лечение проявлений АД на лице. Медианная площадь поражения уменьшилась до 0\% через 1,5 года применения в режиме «по требованию» и сохранялась на этом уровне до конца исследования.

\section{Применение ТКС и ПИМ}

Применение ПИМ было ассоциировано со значительным стероидсберегающим эффректом. В группе ПИМ 36\% пациентов не применяли ТКС вообще. За 5 лет пациенты в группе ПИМ применяли ТКС только 7 дней (медиана) (Q1: 0, Q3: 49 дней) по сравнению со 178 днями (Q1: 77, Q3: 396 дней) в группе TKC. Медианная продолжительность применения ПИМ составила 224,5 дня (Q1: 90, Q3: 452 дней).

\section{Безопасность}

В обеих терапевтических группах наиболее часто отмечались распространенные НЯ. Лечение редко прекращали из-за НЯ: в группе ПИМ 0,6\% (чаще всего реакции в месте нанесения у 3 пациентов); в группе TKC 1,0\% (чаще всего телеангиэктазия - 2 пациента). Отсутствовала разница между группами в темпах роста и скорректированной по Каплану - Мейеру ча-

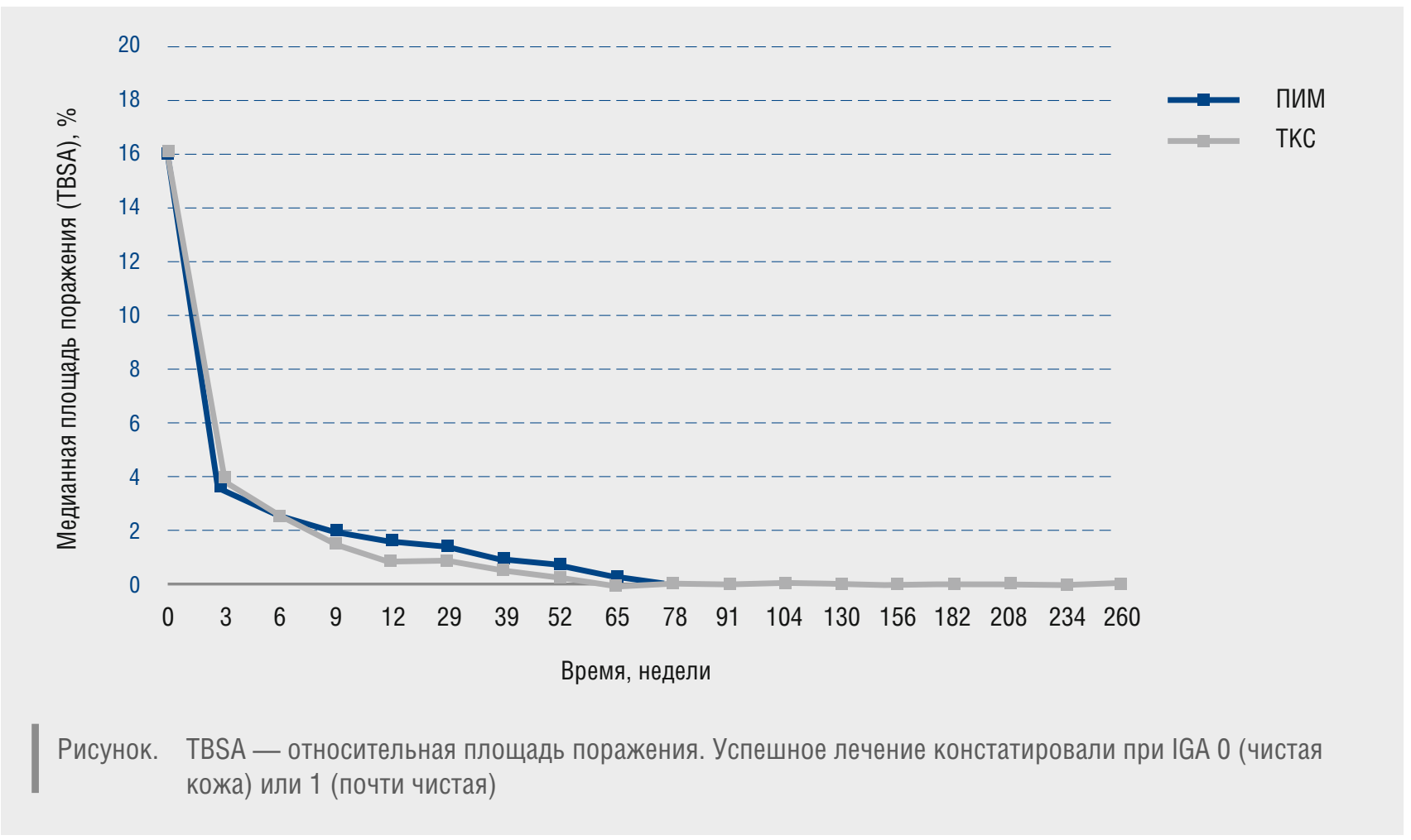


стоте НЯ ( $\geq 5 \%)$ или НЯ, представляющих особый клинический интерес (бактериальные или вирусные инфекции). Анализ с использованием регрессионной модели Пуассона с повторными измерениями показал, что у пациентов в группе ПИМ чаще диагностировали бронхит $(p=0,02)$, инфекционную экзему $(p<0,001)$, импетиго $(p=0,045)$ и назофрарингит $(p=0,04)$. В первые 6 нед. и на протяжении всего исследования отмечались сопоставимые показатели частоты (общий коэффрициент заболеваемости и заболеваемость в пересчете на одного пациента в единицу времени наблюдения НЯ представляют особый клинический интерес). Анализ Каплана - Мейера не выявил разницы во времени до возникновения этих НЯ.

В группе ТКС было зарегистрировано два летальных исхода, но они не были связаны с исследуемым препаратом (утопление, острый лимфоцитарный лейкоз). В двух группах была получена сходная общая частота серьезных НЯ (в группе ПИМ 20,5\%; в группе ТКС 17,3\%) и частота серьезных инфекций и инвазий (13,0\% против 12,4\%). В группе ТКС диагностировали два злокачественных заболевания (острый лимсроцитарный лейкоз, эпендимома), в группе ПИМ была выявлена одна доброкачественная опухоль (пиломатриксома).

\section{Иммунная система}

Титры антител к стандартным вакцинным антигенам у детей, получавших лечение ПИМ или ТKC, были схожими и находились в пределах нормальных значений. Напряженность гуморального иммунитета в ответ на вакцинацию оценивали по титрам антител к антигенам вакцины против Haemophilus influenza типа b до введения третьей дозы вакцины и через 30 дней после завершения иммунизации. В обеих группах все пациенты, кроме одного, были серопозитивны. Повышение уровня иммуноглобулинов и снижение содержания Т- и В-лимфоцитов в периферической крови от исходного значения до 260-й недели было сходным в обеих группах и соответствовало возрастной норме. Доля пациентов с положительным результатом кожного тестирования на грибы рода Candida была сопоставимой в обеих группах (исходно: 14,0\% в группе ПИМ; 9,2\% в группе ТKС; 260-я неделя: 15,3\% против 14,3\%).

Функцию Т-клеток оценивали по продукции цитокинов ex vivo в ответ на стимуляцию анти-CD3 антителами и столбнячным антигеном. По продукции ИЛ-2, ИЛ-4, ИЛ-10 и интерферона- $\gamma$ обе группы были сопоставимы, что указывает на сходство иммунного ответа организма на неспецифическую активацию и специфрические антигены.

\section{Обсуждение}

Безопасность и эффрективность применения ПИМ и ТKC изучали на большой популяции детей с АД с самым длинным сроком наблюдения (т.е. первые 5-6 лет жизни).
Для исследования отбирали детей в возрасте от 3 до 12 мес., чтобы эффрект лечения можно было оценивать на протяжении всего периода младшего детского возраста. Полученные данные указывают на сопоставимую эффективность ПИМ и ТКС в условиях реальной клинической практики, что представляет большой интерес, так как ПИМ на текущий момент не применяется широко в качестве препарата первой линии в терапии АД, поскольку считается менее эффеективным, чем TKC [21]. Оба вида терапии (ПИМ и ТKС) характеризуются быстрым началом действия (в течение 3 нед.). После 5 лет применения препарата в режиме «по требованию» у 88,7\% пациентов в группе ПИМ и 92,3\% в группе ТКС отмечались минимальные проявления АД, что может отражать прогрессирующее повышение эффрективности лечения и/или естественное течение АД с ослаблением клинических проявлений по мере взросления. Быстрое и стойкое улучшение АД может существенно улучшить фризическое и эмоциональное состояние пациента [22]. Полученные результаты подтверждают и расширяют данные предыдущих менее долгосрочных исследований, которые показали, что ПИМ быстро облегчает зуд, эффективно предупреждает рецидивы и увеличивает количество дней ремиссии $[14,15,23,24]$.

Следствием применения ПИМ был значительный стероидсберегающий эффрект. 36\% детей вообще не потребовались TKC за 5 лет исследования. Пациенты в группе ПИМ применяли ТКС только в течение 7 дней (медиана). Имеет большое значение существенное снижение потребности в ТКС. Это демонстрирует, что пациентов с АД можно длительно вести и без кортикостероидов. До 1/3 пациентов не соблюдают режим применения ТKС из-за стероидофобии, связанной с боязнью возникновения побочных эффректов [9, 25-27]. Стероидсберегающий эффрект ПИМ может улучшить приверженность пациентов лечению, хотя специальная оценка этого эффекта в исследовании Petite не проводилась. В этом пятилетнем исследовании не возникло проблем, связанных с безопасностью при применении ПИМ и ТКС. Тип и частота НЯ, в том числе инфекций, соответствовали ожиданиям для этой популяции пациентов $[28,29]$. Во время исследования в группе ПИМ не было случаев возникновения Т-клеточной лимфомы или злокачественных заболеваний кожи, что соответствует данным нескольких долгосрочных клинических исследований $[30,31]$. На протяжении всего исследования в группе ПИМ наблюдалась общая тенденция к более низкому риску возникновения ринореи, вирусной сыпи и инфекций нижних дыхательных путей. Напротив, в другом рандомизированном двойном слепом исследовании с участием взрослых пациентов частота инфекций была выше при применении ТKС [32]. Разница между группами в частоте возникновения НЯ составила всего от 2 до 4\% (без коррекции на множественность 
сравнений). Поэтому клиническое значение наблюдаемых небольших различий в частоте четырех конкретных НЯ следует трактовать с осторожностью.

Иммунологические тесты, проводившиеся в рамках данного исследования, дают самую полную оценку влияния лечения АД на созревание иммунной системы на большой международной популяции детей в первые 5-6 лет жизни. В исследовании не было группы плацебо, что связано с этическими соображениями. Однако в обеих группах отмечено сходное повышение уровня иммуноглобулинов и снижение содержания циркулирующих Т- и В-лимфоцитов, соответствующее динамике созревания иммунной системы у здоровых детей [3338]. Ранее было показано, что у здоровых детей уровень $\lg$ и $\lg$ А прогрессивно повышается в первые годы жизни. Уровень lgЕ также повышается, но в меньшей степени, а уровень IgM выходит на плато раньше, чем уровень других иммуноглобулинов, что соответствует нашим данным [33-36]. Как и в исследованиях других авторов, количество Т- и В-лимфоцитов достигало пика в первые 1-2 года жизни, а затем медленно снижалось до типичных для взрослых значений [37, 38]. Титры поствакцинальных антител подтвердили наличие нормального иммунного ответа на стандартные вакцинные антигены у большинства пациентов, в обеих группах был сходный ответ на вакцинацию. Наши результаты подтверждают, что применение ПИМ у детей в течение 2 лет не приводит к значительному увеличению частоты системных или кожных инфекций и не нарушает иммунный ответ на вакцинацию [14, 15, 19, 23, 39]. Исследование дает важную информацию, полученную в условиях реальной клинической практики, и подтверждает безопасность применения ПИМ у детей младшего возраста с развивающейся иммунной системой. Отсутствие системной иммуносупрессии, вероятно, объясняется минимальным системным воздействием на организм детей грудного и младшего возраста [40, 41].

\section{Выводы}

Таким образом, исследование Petite впервые показало, что применение ПИМ или ТКС в режиме «по требованию» не должно вызывать опасений и не влияет на созревание развивающейся иммунной системы. Полученные данные подтверждают, что ПИМ и ТKC обладают сходной эфрфективностью и что применение ПИМ связано с существенным стероидсберегающим эфффектом. В исследовании получены реальные данные о применении ПИМ в терапии первой линии АД легкой и средней степени тяжести у детей грудного и младшего детского возраста.

\section{Литература}

1. Kubanova A.A., Zaslavsky D.V., Kubanov A.A et al. Atopic dermatitis (Guidelines for management of atopic dermatitis). M 2010; 39. [Кубанова А.А., Заславский Д.В., Кубанов А.А. и др. Атопический дерматит (клинические рекомендации). М, 2010; 39.]

2. Bieber T. Atopic dermatitis. N Engl J Med 2008; 358 (14): 1483-1494.

3. Wadonda-Kabondo N., Sterne J.A., Golding J., Kennedy C.T., Archer C.B., Dunnill M.G. ALSPAC Study Team. A prospective study of the prevalence and incidence of atopic dermatitis in children aged 0 — 42 months. Br J Dermatol 2003; 149 (5): 1023-1028.

4. McKenna S.P., Doward L.C. Quality of life of children with atopic dermatitis and their families. Curr Opin Allergy Clin Immunol 2008; 8 (3): 228-231

5. Spergel J.M., Paller A.S. Atopic dermatitis and the atopic march. J Allergy Clin Immunol 2003; 112 (Suppl 6): S118_S127.

6. Federal clinical guidelines for management of atopic dermatitis (National clinical recommendations).Russian society of dermatovenerologists and cosmetologists. M 2013 [Федеральные клинические рекомендации по ведению пациентов с атопическим дерматитом. (Национальные клинические рекомендации) Российское общество дерматовенерологов и косметологов. М, 2013.]
7. Ring J., Alomar A., Bieber T. et al; European Dermatology Forum (EDF); European Academy of Dermatology and Venereology (EADV); European Federation of Allergy (EFA); European Task Force on Atopic Dermatitis (ETFAD); European Society of Pediatric Dermatology (ESPD); Global Allergy and Asthma European Network (GA2LEN). Guidelines for treatment of atopic eczema (atopic dermatitis) part I. J Eur Acad Dermatol Venereol. 2012; 26 (8): 1045-1060

8. Gorlanov I.A., Zaslavsky D.V., Milyavskaya I.R., Leina L.M., Olovyanishnikov 0.V., Kulikova S.Yu. Pediatric dermatovenereology: Textbook. M: Academy 2012; 352. [Горланов И.А., 3аславский Д.В., Милявская И.Р., Леина Л.М., Оловянишников О.В., Куликова С.Ю. Детская дерматовенерология: Учебник М: Академия 2012; 352.]

9. Aubert-Wastiaux H., Moret L., Le Rhun A. et al. Topical corticosteroid phobia in atopic dermatitis: a study of its nature, origins and frequency. Br J Dermatol 2011; 165 (4): 808-814.

10. Hultsch T., Muller K.D., Meingassner J.G., Grassberger M., Schopf R.E., Knop J. Ascomycin macrolactam derivative SDZ ASM 981 inhibits the release of granuleassociated mediators and of newly synthesized cytokines in RBL $2 \mathrm{H} 3$ mast cells in an immunophilin-dependent manner. Arch Dermatol Res 1998; 290 (9): 501—507.
11. Grassberger M., Baumruker T., Enz A. et al. A novel anti-inflammatory drug, SDZ ASM 981, for the treatment of skin diseases: in vitro pharmacology. Br J Dermatol 1999; 141 (2): 264—273.

12. Queille-Roussel C., Paul C., Duteil L. et al. The new topical ascomycin derivative SDZ ASM 981 does not induce skin atrophy when applied to normal skin for 4 weeks:a randomized, doubleblind controlled study. Br J Dermatol 2001; 144 (3): 507-513

13. Jensen J.M., Pfeiffer S., Witt M. et al. Different effects of pimecrolimus and betamethasone the skin barrier in patients with atopic dermatitis. J Allergy Clin Immunol 2009; 124 (3 suppl 2): R19-R28.

14. Kapp A., Papp K., Bingham A. et al. Flare Reduction in Eczema With Elidel (infants) multicenter investigator study group. Long-term management of atopic dermatitis in infants with topical pimecrolimus, a nonsteroid antiinflammatory drug. J Allergy Clin Immunol 2002; 110 (2): 277-284.

15. H.V.C., Gupta A., Kaufmann R. et al. Safety and efficacy of nonsteroid pimecrolimus cream $1 \%$ in the treatment of atopic dermatitis in infants. J Pediatr 2003; 142 (2): 155-162.

16. Papp K., Staab D., Harper J. et al. Multicentre Investigator Study Group. Effect of pimecrolimus cream $1 \%$ on the long-term course of pediatric atopic dermatitis. Int J Dermatol 2004; 43 (12): 978-983. 
17. Wahn U., Bos J.D., Goodfield M. et al. Flare Reduction in Eczema with Elidel (Children) Multicenter Investigator Study Group. Efficacy and safety of pimecrolimus cream in the long-term management of atopic dermatitis in children. Pediatrics. 2002; 110 (1 pt 1). Available at: www. pediatrics.org/cgi/ content/full/110/1/e2

18. Sigurgeirsson B., H.V., Ferrándiz C., Andriano K., Grinienko A., Jimenez P. Pimecrolimus $1 \%$ cream in (paediatric) Eczema: Prevention of Progression multi-centre investigator study group. Effectiveness and safety of a preventionof-flare-progression strategy with pimecrolimus cream $1 \%$ in the management of paediatric atopic dermatitis. J Eur Acad Dermatol Venereol 2008; 22 (11): 1290-1301.

19. Paul C., Cork M., Rossi A.B., Papp K.A., Barbier N., de Prost Y. Safety and tolerability of $1 \%$ pimecrolimus cream among infants: experience with 1133 patients treated for up to 2 years. Pediatrics. 2006; 117 (1). Available at: www.pediatrics.org/cgi/content/full/117/ 1/e118

20. Sigurgeirsson B., Boznanski A., Todd G. et al. Safety and Efficacy of Pimecrolimus in Atopic Dermatitis: A 5-Year Randomized Trial. PEDIATRICS 2015; 135 (4): 598-606.

21. El-Batawy M.M., Bosseila M.A., Mashaly H.M., Hafez V.S. Topical calcineurin inhibitors in atopic dermatitis: a systematic review and metaanalysis. J Dermatol Sci 2009; 54 (2): 76—87.

22. Lewis-Jones S. Quality of life and childhood atopic dermatitis: the misery of living with childhood eczema. Int J Clin Pract 2006; 60 (8): 984-992.

23. Papp K.A., Werfel T., Fölster-Holst R. et al. Long-term control of atopic dermatitis with pimecrolimus cream $1 \%$ in infants and young children: a two-year study. J Am Acad Dermatol 2005; 52 (2): 240—246.
24. Kaufmann R., Fölster-Holst R., Höger P. et al. CASM981CDE04-Study Group. Onset of action of pimecrolimus cream $1 \%$ in the treatment of atopic eczema in infants. J Allergy Clin Immunol 2004; 114 (5): 1183-1188.

25. Eichenfield L.F., Totri C. Optimizing outcomes for paediatric atopic dermatitis. Br J Dermatol 2014; 170 (suppl 1): 31-37.

26. Charman C.R., Morris A.D., Williams H.C. Topical corticosteroid phobia in patients with atopic eczema. Br J Dermatol 2000; 142 (5): 931-936.

27. Fukaya M. Why do patients with atopic dermatitis refuse to apply topical corticosteroids? Dermatol 2000; 201 (3): 242-245.

28. Pel J.Z. Common infections during the first five years of life. J Hyg (Lond) 1962; 60: 163-174.

29. Posfay-Barbe K.M. Infections in pediatrics: old and new diseases. Swiss Med Wkly 2012; 142 : w13654.

30. Arellano F.M., Wentworth C.E., Arana A., Fernández C., Paul C.F. Risk of lymphoma following exposure to calcineurin inhibitors and topical steroids in patient with atopic dermatitis. J Invest Dermatol 2007; 127 (4): 808_816.

31. Carr W.W. Topical calcineurin inhibitors for atopic dermatitis: review and treatment recommendations. Paediatr Drugs 2013; 15 (4): 303-310.

32. Luger T.A., Lahfa M., Fölster-Holst R. et al. Long-term safety and tolerability of pimecrolimus cream $1 \%$ and topical corticosteroids in adults with moderate to severe atopic dermatitis. J Dermatolog Treat 2004; 15 (3): 169_178.

33. Aksu G., Genel F., Koturoglu G., Kurugöl Z., Kütükçüler N. Serum immunoglobulin (lgG, $\lg \mathrm{M}, \lg A$ ) and $\lg \mathrm{G}$ subclass concentrations in healthy children: a study using nephelometric technique. Turk J Pediatr 2006; 48 (1): 19—24.
34. Jolliff C.R., Cost K.M., Stivrins P.C. et al. Reference intervals for serum $\lg G, \lg A, \lg M, C 3$, and C4 as determined by rate nephelometry. Clin Chem 1982; 28 (1): 126-128.

35. Saarinen U.M., Juntunen K., Kajosaari M., Björkstén F. Serum immunoglobulin E in atopic and non-atopic children aged 6 months to 5 years. A follow-up study. Acta Paediatr Scand 1982; 71 (3): 489—494.

36. Buckley R.H., Dees S.C., O'Fallon W.M. Serum immunoglobulins. I. Levels in normal children and in uncomplicated childhood allergy. Pediatrics 1968; 41 (3): 600—611.

37. Comans-Bitter W.M., de Groot R., van den Beemd R. et al. Im-munophenotyping of blood lymphocytes in childhood. Reference values for lymphocyte subpopulations. J Pediatr 1997; 130 (3): 388-393.

38. Hicks M.J., Jones J.F., Minnich L.L., Weigle K.A., Thies A.C., Layton J.M. Age-related changes in T- and B-lymphocyte subpopulations in the peripheral blood. Arch Pathol Lab Med 1983; 107 (10): 518—523.

39. Papp K.A., Breuer K., Meurer M. et al. Longterm treatment of atopic dermatitis with pimecrolimus cream $1 \%$ in infants does not interfere with the development of protective antibodies after vaccination. J Am Acad Dermatol 2005; 52 (2): 247-253.

40. Langley R.G., Luger T.A., Cork M.J., Schneider D., Paul C. An update on the safety and tolerability of pimecrolimus cream 1\%: evidence from clinical trials and postmarketing surveillance. Dermatology 2007; 215 (suppl 1): 27-44.

41. Lakhanpaul M., Davies T., Allen B.R., Schneider D. Low systemic exposure in infants with atopic dermatitis in a 1-year pharmacokinetic study with pimecrolimus cream 1\%*. Exp Dermatol 2006; 15 (2): 138-141.

об авторе:

Д.В. Заславский - д.м.н., профрессор, профрессор каффедры дерматовенерологии ГБОУ ВПО СПбГПМУ Минздрава России

\section{Конфликт интересов}

Автор заявляет об отсутствии потенциального конфрликта интересов, требующего раскрытия в данной статье 